\title{
COMBINING ABILITY FOR OIL CONTENT AND ITS CORRELATIONS WITHOTHER YIELD COMPONENTS IN SUNFLOWER (Helianthus annuus L.)
}

Hladni, N.. ${ }^{{ }^{*}}$ Škorić, D., ${ }^{1}$ Kraljević-Balalić, M., ${ }^{1,2}$

Sakač, Z., ${ }^{1}$ Jovanović, D. ${ }^{1}$

${ }^{1}$ Institute of Field and Vegetable Crops, Oil Crops Department, 21000 Novi Sad,

Maksima Gorkog 30, Serbia

${ }^{2}$ Faculty of Agriculture, 21000 Novi Sad, Serbia

Received: September 10, 2005

Accepted: March 25, 2006

SUMMARY

Oil yield is a major characteristic of each sunflower hybrid (Škorić et al., 2005). To be able to develop new high-oil sunflower hybrids by the method of interspecific hybridization, it is necessary to have information on mode of inheritance and combining abilities of inbred lines used. When selecting prospective lines as components of future hybrids, it is important to know correlations between yield components on one side and oil content on another.

Seven new divergent cytoplasmic male sterile lines (A) developed by interspecific hybridization, three $R f$-restorer lines used as testers and $21 \mathrm{~F}_{1}$ hybrids have been subjected to the line $\times$ tester analysis. Significant differences have been obtained in mean values for all characteristics under study.

Significant differences were found between A lines and R lines on one side and their $\mathrm{F}_{1}$ hybrids on the other in oil content, plant height, head diameter, total number of seeds per head, 1000-seed weight and seed yield per plant. The line NS-GS-4 exhibited a highly significant positive GCA value for oil content. The line NS-GS-5 had a highly significant negative GCA value for oil content. The hybrid NS-GS-6 $\times$ RHA-R-PL-2/1 had a highly significant positive SCA value for oil content. Non-additive component of genetic variance played the main role in the inheritance of oil content, as indicated by the analysis of variance of combining abilities and the analysis of components of genetic variance. Further confirmation was the ratio GCA/SCA for oil content in the $\mathrm{F}_{1}$ generation which was smaller than unity (0.33). The highest average contribution to the expression of oil content (77.3\%) was exhibited by the A lines. Highly significant negative correlations were found between oil content on one side and head diameter, total number of seed per head, 1000-seed weight and seed yield per plant on the other.

Key words: sunflower, oil content, combining abilities, gene effects, correlations

* Corresponding author, Phone: +381 214898 411, Fax: +381 21413 833,

e-mail: nadah@ifvcns.ns.ac.yu 


\section{INTRODUCTION}

The sunflower (Helianthus annuus L.) is the fourth largest oilcrop, after soybean, oil palm and rapeseed (Fernandez-Martinez et al., 2004).

In Serbia and Montenegro, sunflower is the main crop for production of edible oil.

The main objectives of sunflower breeding are improvements in seed yield and oil content per unit area, sink capacity, harvest index, resistance to dominant diseases and pests, and plant architecture (Škorić et al., 2002). The last parameter should enable to increase stand density, therefore seed and oil yields, under intensive cultivation practices.

Oil content varies with location (38.1-49.2\%) (Škorić et al., 1996) and year of growing (36.0-54.4\%) (Škorić and Marinković, 1990).

Oil content in seed is greatly affected by genotype, soil and climatic conditions and the intensity of cultivation practices (Marinković et al., 2003).

Cultivated sunflower exhibits considerable variability in oil content. In general, however, oil content is much lower in wild species than in cultivated sunflower (Seiler, 1992).

For development of new, high-oil and stable sunflower hybrids by the method of interspecific hybridization, it is necessary to gain information on mode of inheritance and combining abilities of prospective inbred lines to be used as components of future hybrids.

High and low combining abilities of sunflower inbred lines are frequent topic for discussion. It has been proved experimentally that lines with high GCA produce higher yielding hybrids than lines with low GCA (Marinković, 1993; Joksimović et al. 1993).

Genetic distance between parent lines is a prerequisite for expression of high SCA (Škorić et al., 2004).

Control of inheritance of oil content by additive genes has been observed by Škorić (1976), Miller et al. (1980), Marinković (1984), Ortegon-Morales et al. (1992), Rojas and Fernandez-Martinez (1998), Ashok et al. (2000), etc. Kovačik and Škaloud (1972), Marinković (1993), Škorić et al. (2000) and Parameswari et al. (2004) reported that the inheritance of oil content is controlled by non-additive genes.

Highly significant positive correlations have been observed between oil content in seed on one side and head diameter, number of seeds per head and 1000-seed weight on the other (Marinković and Škorić, 1988; Marinković et al, 1994).

Positive direct effect of oil content on seed yield has been observed by Chaudhary and Anand (1993) and Razi et al, (1999).

Objectives of this study were to assess the effects of general combining ability (GCA) of inbred lines and specific combining ability (SCA) of $F_{1}$ hybrids, gene effects, components of genetic variance and average contributions (\%) of lines, test- 
ers and their interactions on expression of oil content in sunflower seed. Further objectives were to establish mutual relationships between plant height, head diameter, total number of seeds per head, 1000-seed weight and seed yield per plant on one side and oil content on the other.

\section{MATERIAL AND METHOD}

Seven new divergent (A) cytoplasmic male sterile inbred lines, three $R f$-restorer lines and $21 \mathrm{~F}_{1}$ hybrids, all developed at Institute of Field and Vegetable Crops in Novi Sad, were used in this study. The female lines (NS-GS-1, NS-GS-2, NS-GS-3, NS-GS-4, NS-GS-5, NS-GS-6, NS-GS-7) had been developed by interspecific hybridization. The male lines (RHA-R-PL-2/1, RHA-N-49, RUS-RF-OL-168) with high combining abilities and in the form of fertility restorers were used as testers. All testers were crossed with all female lines to produce the $\mathrm{F}_{1}$ generation.

The trial was established at Rimski Šančevi experiment field of Institute of Field and Vegetable Crops, following random block design in three replications. The lines and hybrids were planted by hand, at optimum planting date and into a carefully prepared seedbed. The experimental unit included six rows, with 12 plants per row. The distance between rows was $70 \mathrm{~cm}, 25 \mathrm{~cm}$ in the row. The basic sample consisted of 30 plants ( 10 plants per replication), taken from inner rows in each plot. Plant height (a distance from soil surface to the middle of the head) and head diameter $(\mathrm{cm})$ were measured at the stage of physiological maturity. Seed yield per plant was measured after harvest in the laboratory - seed obtained in open pollination were weighed on a technical scale with an accuracy of $0.01 \mathrm{~g}$. The weight of 1000 seeds was determined in random samples of absolutely clean and air-dry seed. Total number of seeds per head was determined by counting well-filled seeds on the head. Oil content in seed was analyzed in an NMR analyzer, in the chemical laboratory of Oilcrops Department of Institute of Field and Vegetable Crops.

Determination of mean values and correlation coefficients $(r)$ as indicators of mutual dependence were conducted according to Hadživuković (1991). The analysis of combining abilities was conducted according to the line $\times$ tester method (Singh and Choudhary, 1976).

\section{RESULTS AND DISCUSSION}

The tested $A$ lines, $R$ lines and their $F_{1}$ hybrids exhibited significant differences in their mean values for oil content, plant height, head diameter, total number of seed per head, 1000-seed weight and seed yield per plant (Table 1). The inbred line NS-GS-4 showed a highly significant positive GCA value for oil content, the line NSGS-5 a highly significant negative GCA value (Table 2). The highest positive and negative SCA values for oil content were exhibited by the hybrids NS-GS- $6 \times$ RHA-RPL-2/1 and NS-GS-5 × RHA-R-PL-2/1, respectively (Table 3). 
Table 1: Mean values for plant height, head diameter, total number of seed per head, 1000seed weight, seed yield per plant and oil content in sunflower seed

\begin{tabular}{|c|c|c|c|c|c|c|c|}
\hline \multirow{3}{*}{ No. } & \multirow{3}{*}{$\begin{array}{l}\text { Parents and } \\
\text { hybrids }\end{array}$} & VB & $\overline{P G}$ & UBsg & $m-1000$ & PS & SU \\
\hline & & $\mathrm{cm}$ & $\mathrm{cm}$ & & $g$ & $g$ & $\%$ \\
\hline & & $\bar{x} \pm s_{\bar{x}}$ & $\bar{x} \pm s_{\bar{x}}$ & $\bar{x} \pm s_{\bar{x}}$ & $\bar{x} \pm s_{\bar{x}}$ & $\bar{x} \pm s_{\bar{x}}$ & $\bar{x} \pm s_{\bar{x}}$ \\
\hline$\overline{1}$ & NS-GS-1 & $92.8 \pm 0.50$ & $20.1 \pm 0.64$ & $1032.9 \pm 8.21$ & $49.2 \pm 0.74$ & $35.6 \pm 1.46$ & $46.3 \pm 0.49$ \\
\hline 2 & NS-GS-2 & $105.9 \pm 0.61$ & $20.3 \pm 0.11$ & $1081.0 \pm 18.79$ & $54.2 \pm 0.83$ & $52.8 \pm 1.79$ & $49.9 \pm 0.49$ \\
\hline 3 & NS-GS-3 & $117.2 \pm 0.70$ & $19.6 \pm 0.10$ & $939.5 \pm 28.53$ & $52.9 \pm 1.08$ & $50.5 \pm 1.25$ & $43.1 \pm 0.33$ \\
\hline 4 & NS-GS-4 & $105.7 \pm 0.53$ & $21.3 \pm 0.16$ & $620.0 \pm 15.49$ & $94.8 \pm 2.21$ & $55.4 \pm 2.31$ & $29.5 \pm 0.48$ \\
\hline 5 & NS-GS-5 & $85.8 \pm 0.54$ & $21.7 \pm 0.10$ & $709.2 \pm 17.61$ & $79.4 \pm 1.27$ & $57.0 \pm 1.50$ & $38.1 \pm 0.47$ \\
\hline 6 & NS-GS-6 & $68.0 \pm 0.49$ & $21.6 \pm 0.14$ & $698.8 \pm 25.46$ & $51.9 \pm 0.96$ & $32.4 \pm 1.65$ & $37.0 \pm 0.37$ \\
\hline 7 & NS-GS-7 & $86.7 \pm 0.53$ & $21.4 \pm 0.11$ & $874.6 \pm 32.99$ & $44.3 \pm 0.79$ & $43.8 \pm 1.75$ & $43.1 \pm 0.55$ \\
\hline 8 & RHA-R-PL-2/1 & $125.2 \pm 0.60$ & $16.1 \pm 0.15$ & $614.0 \pm 21.00$ & $49.8 \pm 0.57$ & $30.1 \pm 1.19$ & $49.5 \pm 0.33$ \\
\hline 9 & RHA-N-49 & $111.4 \pm 0.79$ & $12.2 \pm 0.75$ & $806.0 \pm 29.51$ & $27.6 \pm 0.50$ & $23.7 \pm 1.08$ & $51.3 \pm 0.27$ \\
\hline 10 & RUS-RF-OL-168 & $127.0 \pm 0.70$ & $16.5 \pm 0.15$ & $968.5 \pm 23.68$ & $29.4 \pm 0.38$ & $25.5 \pm 0.86$ & $49.3 \pm 0.29$ \\
\hline$\overline{11}$ & $1 \times 8$ & $149.3 \pm 0.74$ & $21.9 \pm 0.14$ & $1652.6 \pm 50.01$ & $50.2 \pm 0.85$ & $79.6 \pm 2.42$ & $49.5 \pm 0.47$ \\
\hline 12 & $1 \times 9$ & $159.3 \pm 0.66$ & $21.7 \pm 0.18$ & $1995.5 \pm 61.98$ & $45.8 \pm 0.82$ & $91.8 \pm 3.48$ & $52.2 \pm 0.45$ \\
\hline 13 & $1 \times 10$ & $135.3 \pm 1.00$ & $23.0 \pm 0.15$ & $1903.2 \pm 54.62$ & $49.7 \pm 0.84$ & $96.6 \pm 2.71$ & $51.0 \pm 0.39$ \\
\hline 14 & $2 \times 8$ & $154.2 \pm 0.95$ & $22.8 \pm 0.17$ & $1595.5 \pm 38.18$ & $51.7 \pm 0.93$ & $82.2 \pm 2.65$ & $49.2 \pm 0.49$ \\
\hline 15 & $2 \times 9$ & $154.8 \pm 0.69$ & $23.0 \pm 0.15$ & $2089.6 \pm 59.58$ & $47.4 \pm 0.71$ & $96.9 \pm 2.54$ & $50.2 \pm 0.37$ \\
\hline 16 & $2 \times 10$ & $138.5 \pm 0.79$ & $23.1 \pm 0.12$ & $1737.9 \pm 47.23$ & $48.0 \pm 1.09$ & $81.7 \pm 2.49$ & $49.8 \pm 0.54$ \\
\hline 17 & $3 \times 8$ & $168.8 \pm 0.65$ & $23.8 \pm 0.15$ & $1597.7 \pm 27.02$ & $58.0 \pm 0.80$ & $89.9 \pm 1.39$ & $47.7 \pm 0.41$ \\
\hline 18 & $3 \times 9$ & $177.7 \pm 0.99$ & $23.5 \pm 0.17$ & $2120.7 \pm 50.89$ & $50.8 \pm 1.17$ & $106.2 \pm 2.65$ & $48.3 \pm 0.33$ \\
\hline 19 & $3 \times 10$ & $156.2 \pm 1.10$ & $24.9 \pm 0.14$ & $1791.7 \pm 36.67$ & $54.6 \pm 1.10$ & $102.0 \pm 2.74$ & $46.5 \pm 0.49$ \\
\hline 20 & $4 \times 8$ & $161.3 \pm 0.66$ & $23.8 \pm 0.19$ & $1559.2 \pm 64.03$ & $78.6 \pm 1.11$ & $111.1 \pm 2.67$ & $43.4 \pm 0.47$ \\
\hline 21 & $4 \times 9$ & $164.0 \pm 0.64$ & $23.0 \pm 0.19$ & $1695.5 \pm 38.67$ & $54.6 \pm 0.76$ & $94.4 \pm 2.68$ & $46.7 \pm 0.24$ \\
\hline 22 & $4 \times 10$ & $159.0 \pm 0.72$ & $24.0 \pm 0.22$ & $1521.4 \pm 37.50$ & $65.5 \pm 1.22$ & $103.3 \pm 1.81$ & $45.8 \pm 0.36$ \\
\hline 23 & $5 \times 8$ & $149.8 \pm 1.69$ & $29.2 \pm 0.31$ & $2009.0 \pm 44.26$ & $85.7 \pm 1.85$ & $162.9 \pm 3.28$ & $41.5 \pm 0.65$ \\
\hline 24 & $5 \times 9$ & $163.7 \pm 1.29$ & $24.6 \pm 0.22$ & $2025.7 \pm 72.78$ & $64.7 \pm 1.08$ & $117.0 \pm 3.71$ & $45.9 \pm 0.59$ \\
\hline 25 & $5 \times 10$ & $149.0 \pm 1.14$ & $24.8 \pm 0.13$ & $1519.3 \pm 38.78$ & $68.8 \pm 1.16$ & $112.4 \pm 2.40$ & $45.4 \pm 0.32$ \\
\hline 26 & $6 \times 8$ & $137.5 \pm 0.99$ & $24.7 \pm 0.23$ & $1390.0 \pm 61.82$ & $51.8 \pm 1.19$ & $79.0 \pm 3.18$ & $47.6 \pm 0.61$ \\
\hline 27 & $6 \times 9$ & $131.3 \pm 1.05$ & $29.5 \pm 0.30$ & $2262.6 \pm 69.83$ & $50.8 \pm 0.98$ & $104.7 \pm 2.49$ & $45.7 \pm 0.71$ \\
\hline 28 & $6 \times 10$ & $122.0 \pm 0.90$ & $28.5 \pm 0.21$ & $1715.7 \pm 54.71$ & $52.9 \pm 0.93$ & $87.4 \pm 2.45$ & $46.8 \pm 0.69$ \\
\hline 29 & $7 \times 8$ & $138.5 \pm 0.79$ & $26.9 \pm 0.24$ & $1699.5 \pm 39.55$ & $53.3 \pm 0.93$ & $93.0 \pm 2.09$ & $46.7 \pm 0.46$ \\
\hline 30 & $7 \times 9$ & $139.3 \pm 0.81$ & $29.1 \pm 0.23$ & $2071.1 \pm 69.06$ & $53.5 \pm 0.96$ & $100.4 \pm 2.23$ & $45.9 \pm 0.68$ \\
\hline 31 & $7 \times 10$ & $134.7 \pm 0.70$ & $25.9 \pm 0.18$ & $1676.4 \pm 43.97$ & $53.1 \pm 1.01$ & $94.9 \pm 1.65$ & $48.5 \pm 0.34$ \\
\hline$\overline{\text { LSD }}$ & 0.05 & 1.99 & 0.45 & 99.72 & 2.72 & 3.16 & 1.49 \\
\hline LSD & 0.01 & 2.99 & 0.67 & 149.58 & 4.08 & 4.74 & 2.24 \\
\hline
\end{tabular}

VB-plant height; PG-head diameter; UBSG-total number of seed per head

M 1000-1000-seed weight; PS-seed yield per plant; SU-oil content in seed 
Table 2: GCA values for oil content in seed in sunflower inbred lines

\begin{tabular}{lcc}
\hline No. & Parents & Oil content \\
\hline 1 & NS-GS-1 & $3.56^{\star \star}$ \\
2 & NS-GS-2 & $2.38^{\star \star}$ \\
3 & NS-GS-3 & 0.15 \\
4 & NS-GS-4 & $45.28^{\star *}$ \\
5 & NS-GS-5 & $-3.10^{\star \star}$ \\
6 & NS-GS-6 & $-0.64^{\star}$ \\
7 & NS-GS-7 & -0.30 \\
8 & RHA-R-PL-2/1 & $-0.82^{\star}$ \\
9 & RHA-N-49 & 0.49 \\
10 & RUS-RF-OL-168 & 0.33 \\
\hline SE GCA/lines & 0.43 \\
SE (GCA $A_{i}-$ GCA $) /$ lines & 0.61 \\
SE GCA/testers & 0.28 \\
SE (GCA $A_{i}-$ GCA $) /$ testers & 0.40 \\
LSD (1-7) 5\% & 0.43 \\
LSD (1-7) 1\% & 0.86 \\
LSD (8-10) 5\% & 0.56 \\
LSD (8-10) 1\% & 0.85 \\
\hline
\end{tabular}

As the ratio GCA/SCA (0.33) was lower than 1, it may be concluded that the non-additive gene action (dominance and epistasis) played an important role in the inheritance of oil yield (Table 4). Prevalence of non-additive genetic variance in the inheritance of oil content has been reported by Marinković (1993) and Škorić et al. (2000), while Miller et al. (1980), Marinković (1984), Marinković et al. (2000), Ortegon-Morales et al. (1992), and Rojas and Fernandez-Martinez (1998) reported the prevalence of the additive component. The A lines had the highest contribution to the expression of oil content77.3\% (Table 5). These results are in agreement with those of Škorić et al. (2000) who reported an average contribution of a female line to the expression of oil content amounting to $58.2 \%$, but they are contrary to those of Marinković et al. (2000) who reported that the contribution of restorers, amounting to $52.24 \%$, was more significant.

Highly significant negative relationships were found between oil content on one side and head diameter, total number of seeds per head, 1000-seed weight and seed yield per plant. Highly significant positive correlations were established between seed yield on one side and head diameter and 1000-seed weight on the
Table 3: SCA values for oil content in seed in

\begin{tabular}{lcc}
\hline No. & $\mathrm{F}_{1}$ hybrid & Oil content \\
\hline 1 & $1 \times 8$ & -0.60 \\
2 & $2 \times 8$ & 0.31 \\
3 & $3 \times 8$ & 1.10 \\
4 & $4 \times 8$ & -1.07 \\
5 & $5 \times 8$ & -1.95 \\
6 & $6 \times 8$ & $1.73^{*}$ \\
7 & $7 \times 8$ & 0.52 \\
8 & $1 \times 9$ & 0.84 \\
9 & $2 \times 9$ & -0.02 \\
10 & $3 \times 9$ & 0.26 \\
11 & $4 \times 9$ & 0.91 \\
12 & $5 \times 9$ & 1.16 \\
13 & $6 \times 9$ & $-1.53^{*}$ \\
14 & $7 \times 9$ & $-1.63^{*}$ \\
15 & $1 \times 10$ & -0.24 \\
16 & $2 \times 10$ & -0.29 \\
17 & $3 \times 10$ & -1.32 \\
18 & $4 \times 10$ & 0.16 \\
19 & $5 \times 10$ & 0.79 \\
20 & $6 \times 10$ & -0.20 \\
21 & $7 \times 10$ & 1.12 \\
\hline SE SCA & & 0.282 \\
SE $\left(\mathrm{S}_{\mathrm{ij}}-\mathrm{S}_{\mathrm{ki}}\right)$ & & 0.399 \\
LSD 0.05 & & 1.49 \\
LSD 0.01 & & 2.24 \\
\hline & &
\end{tabular}


Table 4: Components of genetic variance for oil content in sunflower seed

\begin{tabular}{lc}
\hline Component & Oil content \\
\hline GCA & 0.41 \\
$F=0 V_{A}$ & 1.64 \\
$F=1 V_{A}$ & 0.82 \\
$F=0 V_{D} / V_{A}$ & 2.98 \\
$F=0 V_{D} / V_{A}$ & 1.48 \\
SCA & 1.23 \\
$F=0 V_{D}$ & 4.90 \\
$F=1 V_{D}$ & 1.23 \\
GCA $/ S C A$ & 0.33 \\
\hline
\end{tabular}

Table 5: Average contribution (\%) of female lines, testers and their interactions to the expression of oil content in sunflower seed

\begin{tabular}{lc}
\hline \multirow{2}{*}{ Average contribution } & Oil content \\
\cline { 2 - 2 } & $\%$ \\
\hline Females & 77.29 \\
Testers & 5.65 \\
Line $\times$ tester & 17.06 \\
\hline
\end{tabular}

other, $0.448^{* *}$ and $0.805^{* *}$, respectively. A significant correlation was found between seed yield and total number of seeds per head, 0.376*. A significant positive correlation was also found between head diameter and total number of seeds per head, 0.329*, respectively (Table 6).

Table 6: Correlation coefficients between the five components of oil content in sunflower seed

\begin{tabular}{lcccccc}
\hline \multirow{2}{*}{ Characteristic } & & $\mathrm{PG}$ & UBSG & $\mathrm{M}-1000$ & $\mathrm{PS}$ & $\mathrm{SU}$ \\
\cline { 3 - 7 } & & $\mathrm{X}_{2}$ & $\mathrm{X}_{3}$ & $\mathrm{X}_{4}$ & $\mathrm{X}_{5}$ & $\mathrm{Y}$ \\
\hline VB & $\mathrm{X}_{1}$ & $-0.543^{\star *}$ & -0.136 & $0.332^{\star}$ & $0.278^{\star}$ & -0.113 \\
PG & $\mathrm{X}_{2}$ & & $0.329^{\star}$ & 0.292 & $0.448^{\star \star}$ & $-0.636^{\star *}$ \\
UBSG & $\mathrm{X}_{3}$ & & & -0.173 & $0.376^{\star}$ & $-0.561^{\star \star}$ \\
M-1000 & $\mathrm{X}_{4}$ & & & & $0.805^{\star \star}$ & $-0.849^{\star *}$ \\
PS & $\mathrm{X}_{5}$ & & & & & $-0.717^{\star \star}$
\end{tabular}

$\mathrm{X}_{1}$ - plant height (VB); $\mathrm{X}_{2}$ - head diameter (PG); $\mathrm{X}_{3}$ - total number of seeds per head (UBSG);

$\mathrm{X}_{4}$-1000-seed weight (M-1000); $\mathrm{X}_{5}$ - seed yield (PS); $\mathrm{Y}$ - oil content in seed

Highly significant relations between seed yield and head diameter have been reported by Škorić (1975), Marinković (1992) and Hladni et al. (2004).

Total number of seed per head and 1000-seed weight had high positive effects on sunflower seed yield (Dušanić et al., 2004).

This and similar studies may be of importance in the development of new highoil sunflower genotypes on the basis of interspecific hybridization.

\section{CONCLUSION}

Significant differences were obtained for the tested genotypes (inbred lines and hybrids) in mean values of all characteristics under study.

The non-additive component of genetic variance played the main role in the inheritance of oil content, as indicated by the analysis of variance of combining abilities and the analysis of components of genetic variance. This was further confirmed 
by the GCA/SCA ratio for oil content in the $\mathrm{F}_{1}$ generation which was smaller than unity (0.33).

The A lines had the highest average contribution to the expression of oil content, $77.3 \%$.

Highly significant negative correlations were found between oil content on one side and head diameter, total number of seed per head, 1000-seed weight and seed yield per plant on the other. Highly significant positive correlations were found between seed yield on one side and head diameter and 1000-seed weight on the other, $0.448^{* *}$ and $0.805^{* *}$, respectively. A significant positive correlation was found between seed yield and total number of seeds per head, 0.376*. A significant positive correlation was also found between head diameter and total number of seeds per head, 0.329*.

\section{REFERENCES}

Ashok, S., Mohamed Sheriff, N., Narayanan, S.L., (2000). Combining ability studies in sunflower (Helianthus annuus L.). Crop Research (Hisar) 20(3): 457-462.

Chaudhary, S.K. and Anand, I.J., (1993). Correlation and path-coefficient analysis in $\mathrm{F}_{1}$ and $\mathrm{F}_{2}$ generations in sunflower (Helianthus annuus L.). International Journal of Tropical Agriculture 11(3): 204-208.

Dušanić, N., Miklič, V., Joksimović, J., Atlagić, J., (2004). Path coefficient analysis of some yield components of sunflower. Proc. of the $16^{\text {th }}$ Inter. Sunf. Conf., Fargo, North Dakota, USA, II, pp. 531-537.

Hadživuković, S., 1991. Statistički metodi. Drugo prošireno izdanje. Poljoprivredni fakultet, Novi Sad.

Hladni, N., Škorić, D., Kraljević-Balalić, M., Ivanović, M., Sakač, Z., Jovanović, D., (2004). Correlation of yield components and seed yield per plant in sunflower (Helianthus annuus L. ). Proc. of the $16^{\text {th }}$ Inter. Sunf. Conf., II, pp. 491-496, Fargo, North Dakota, USA.

Fernández-Martínez, J., Velasco, L., Pérez-Vich, B., (2004). Progress in the genetic modification of sunflower oil quality. Proc. of the $16^{\text {th }}$ Inter. Sunf. Conf., Fargo, North Dakota, USA, II, pp. 1-14.

Joksimović, J., Marinković, R., Mihaljčević, M., (1993). Uticaj lisne površine na prinos semena i ulja kod $F_{1}$ hibrida suncokreta (Helianthus annuus L.). Izvodi saopštenja sa $X$ Simpozijuma jugoslovenskog društva za fiziologiju biljaka, Zemun Polje, Beograd, 22: $17-18$

Kovačik, A., Škaloud, V., (1972). Combining ability and prediction of heterosis in sunflower (Helianthus annuus L.). Sci. Agriculture Bohemoslovaca, XX(4): 263-273.

Marinković, R. (1984). Način nasleđivanja prinosa semena i nekih komponenti prinosa ukrštanjem raznih inbred linija. Doktorska disertacija, Univerzitet u Novom Sadu, Poljoprivredni fakultet.

Marinković, R., Škorić, D., (1988). Path-coefficient analysis of components of sunflower seed yield (H. annuus L.). Proc. of the $12^{\text {th }}$ Inter. Sunf. Conf., Novi Sad, Yugoslavia, pp. 406407.

Marinković, R. (1992). Path-coeficient analysis of some yield components of sunflower (Helianthus annuus L.). Euphytica 60: 201-205.

Marinković, R.. (1993). Combining ability of some inbred sunflower (Helianthus annuus L.) lines. Indian J. Genet. 53: 299-304.

Marinković, R., Škorić, D., Nenadić, N., Jovanović, D., Miklič, V., Joksimović, J., Stanojević, D., Nedeljković, S., (1994). Uticaj položaja semena u glavi na prinos i neke komponente prinosa semena kod suncokreta (H. annuus L.). Zbornik radova Instituta za ratarstvo i povrtarstvo-Novi Sad 22: 379-389.

Marinković, R., Dozet, B. and Vasić, D., (2003). Oplemenjivanje suncokreta (Monografija), Školska knjiga, Novi Sad, pp. 1-368. 
Miller, J.F., Hammond, J.J., Roath, W.W., (1980). Comparison of inbred vs. single cross testers and estimation of genetic effects in sunflower. Crop Sci. 20: 703-706.

Ortegon-Morales, A.S., Escobedo-Mendoza, A., Quilantan-Villarreal, L., (1992). Combining ability of sunflower (Helianthus annuus L.) lines and comparison among parent lines and hybrids. Proc. of the $13^{\text {th }}$ Inter. Sunf. Conf. Pisa, Italy, II: 1178-1193.

Parameswari, C., Muralidharan, V., Subbalakshmi, B., Manivannam, N., (2004). Genetic analysis yield and inportant traits in sunflower (Helianthus annuus L.). Hybrids. Journal of Oil seed Research 21(1): 168-170.

Razi, H., Assad, M.T., (1999). Comparison of selection criteria in normal and limited irrigtion in sunflower. Euphytica 105: 83-90.

Rojas, P. and Fernández-Martínez, J.M., (1998). Combining ability of oil and protein among six sunflower lines. Proc. of the EUCARPIA-International Symposium on Breeding of Protein and Oil Crops, Pontevedra, Spain 117-118: 1-4,

Seiler, G.J., (1992). Utilization of wild sunflower species for the improvement of cultivated sunflower. Field Crops. Res. 30: 195-230.

Singh, R.K. and Choudhary, B.D., (1976). Biometrical Techniques in Genetics and Breeding. Int. Bioscience Publishers. Hisar, India

Škorić, D., (1975). Mogućnost korišćenja heterozisa na bazi muške sterilnosti kod suncokreta. Doktorska disertacija, Univerzitet u Novom Sadu, Poljoprivredni fakultet.

Škorić, D., (1976). Harakter nasledovanija soderžanija masla v semenah pervovo pokolenija i komponenti genetičeskoj izmenčivosti podsolnečnika. Materialj VII Meždunarodnoj konferencii po podsolnečniku, Krasnodar, SSSR, pp. 191-195.

Škorić, D. i Marinković, R., (1990). Stanje u oplemenjivanju i aktuelna problematika u proizvodnji suncokreta. Zbornik radova sa savetovanja o unapređenju uljarstva Jugoslavije, Herceg Novi, pp. 1-15.

Škorić, D., Mihaljčević, M., Jocić, S., Marinković, R., Dozet, B., Atlagić, J., Hladni, N., (1996). Najnovija dostignuća u oplemenjivanju suncokreta. Zbornik radova sa 37 Savetovanja o proizvodnji i preradi uljarica, Budva, pp. 18-25.

Škorić, D., Jocić, S., Molnar, I., (2000). General (GCA) and specific (SCA) combining abilities in sunflower. Proc. of the $15^{\text {th }}$ Int. Sunf. Conf., Toulouse, France, pp. 23-30.

Škorić, D., Marinković, R., Jocić, S., Jovanović, D., Hladni, N., (2002). Dostignuća i dalji pravci u oplemenjivanju suncokreta i izbor hibrida za setvu u 2002 godini. Zbornik radova Naučnog instituta za ratarsvo i povrtarstvo, Sveska 36: 147-160.

Škorić, D., Jocić, S., Jovanović, D., Hladni, N., (2004). Global sunflower breeding achievements. XXXIX Znanstveni skup hrvatskih agronoma sa međunarodnim sudjelovanjem, Agronomski fakultet Zagreb, pp. 172-174.

Škorić, D., Joksimović, J., Jocić, S., Jovanović, D., Marinković, R., Hladni, N., Gvozdenović, S., (2005). Ocena vrednosti produktivnih svojstava NS-hibrida suncokreta. Zbornik radova Naučnog instituta za ratarstvo i povrtarstvo, 41: 21-33.

\section{HABILIDADES DE COMBINACIÓN PARA EL CONTENIDO DE ACEITE Y SUS CORRELACIONES CON OTRAS COMPONENTES DE RENDIMIENTO DE GIRASOL (Helianthus annuus L.)}

\section{RESUMEN}

El rendimiento del aceite es el índice principal de cada híbrido de girasol (te oleosos, sobre la base de interespecies de hibridización, requiere disposición de la información sobre el modo de heredar y las habilidades de combinación de las líneas consanguíneas creadas. También tiene significado la investigación de la mutua independencia del rendimiento y las componentes de rendimiento con el contenido de aceite para elegir las líneas con perspectiva, que se sigan utilizando como componentes de los futuros híbridos de girasol. 
Para el análisis línea x téster, se utilizaron siete nuevas líneas consanguíneas de esterilidad citoplasmática divergentes (A), engendradas por medio de la hibridización de interespecies, tres líneas restauradoras Rf como tésters y 21 híbridos de la generación $F_{1}$. Se obtuvieron significantes diferencias en los valores medios para todas las características investigadas.

Entre las investigadas líneas A y los tésters $R f$ y sus híbridos $\mathrm{F}_{1}$, fueron determinadas significantes diferencias en el contenido de aceite, altura de la planta, diámetro del capítulo, el número total de semilla por capítulo, masa de 1000 semillas y el rendimiento de la semilla por planta. Los valores positivos, altamente significantes de GCA para el contenido de aceite, mostró la línea consanguínea NS-GS-4, mientras que el valor negativo altamente significante de GCA, tuvo la línea consanguínea NS-GS-5. El valor positivo más alto significante de SCA para el contenido de aceite, ha demostrado el híbrido NS-GS-6 $\times$ RHA-R-PL-2/1. El papel principal en la herencia del contenido de aceite, tiene la componente no aditiva de la variancia genética, lo que se ve del análisis de variancias de las habilidades para combinación y análisis de los componentes de la variancia genética. Eso lo confirma también, la relación GCA/SCA para el contenido de aceite $(0.33)$ en la generación $\mathrm{F}_{1}$, cuyo valor es menor de uno. El mayor rendimiento promedio en la expresión del contenido de aceite $(77.3 \%)$ tuvieron las líneas maternas A. La interdependencia negativa altamente significante fue determinada entre el contenido de aceite y el diámetro del capítulo, el número total de semilla por capítulo, masa de 1000 semillas y el rendimiento de la semilla por planta.

\title{
APTITUDE COMBINATOIRE POUR LE CONTENU D'HUILE ET CORRÉLATIONS AVEC D'AUTRES COMPOSANTES DU RENDEMENT CHEZ LE TOURNESOL (Helianthus annuus L.)
}

\author{
RÉSUMÉ
}

Le rendement d'huile est une caractéristique majeure de tout hybride de tournesol (Škorić et al., 2005). Pour pouvoir développer de nouveaux hybrides de tournesol à haute teneur d'huile par la méthode d'hybridation entre espèces, il faut avoir de l'information sur le mode d'hérédité et les aptitudes combinatoires des lignées autogames utilisées. Dans la sélection de lignées potentielles en tant que composantes de futurs hybrides, il est important de connaître les corrélations entre les composantes de rendement d'une part et le contenu d'huile d'autre part.

Sept nouvelles lignées (A) mâles stériles cytoplasmiques divergentes développées par hybridation entre espèces, trois lignées restauratrices Rf utilisées comme contrôles et 21 hybrides $\mathrm{F}_{1}$ ont été soumis à l'analyse lignée $\mathbf{x}$ contrôle. Des différences significatives ont été obtenues dans les valeurs moyennes pour toutes les caractéristiques observées.

Des différences significatives ont été constatées entre les lignées A et $\mathrm{R}$ d'une part et leurs hybrides $\mathrm{F}_{1}$ d'autre part pour le contenu d'huile, la hauteur de la plante, le diamètre de la tête, le nombre total de graines par tête, le poids de 1000 graines et le rendement de graines par plante. La lignée NS-GS-4 a montré une valeur GCA positive très significative pour le contenu d'huile. La lignée NS-GS-5 avait une valeur GCA négative très significative pour le contenu d'huile. L'hybride NS-GS- $6 \times$ RHA-R-PL-2/1 avait une valeur SCA positive très significative pour le contenu d'huile. La composante de variance génétique non 
additive a joué le rôle principal dans l'hérédité du contenu d'huile comme le montre l'analyse de variance des aptitudes combinatoires et l'analyse des composantes de variance génétique. Une confirmation ultérieure était apportée par la proportion CGA/SCA pour le contenu d'huile dans la génération $\mathrm{F}_{1}$ qui était plus petite que l'unité $(0,33)$. La contribution moyenne à l'expression du contenu d'huile le plus élevé $(77,3 \%)$ était manifesté par les lignées A. Des corrélations négatives hautement significatives ont été trouvées entre le contenu d'huile et le diamètre de la tête, le nombre total de graines par tête, le poids de 1000 graines et le rendement de graines par plante. 